\title{
IDEALS AND FILTERS OF PSEUDO-COMPLEMENTED SEMILATTICES
}

\author{
by T. S. BLYTH
}

(Received 15th March 1979)

\section{Introduction}

W. H. Cornish (2) has investigated congruences on pseudo-complemented distributive lattices and has identified those ideals (resp. filters) that are congruence kernels (resp. cokernels). In this paper we show that many of the principal results concerning congruence kernels and cokernels hold in a semilattice and therefore do not depend on distributivity, nor on the existence of unions.

If $(L, \wedge, *, 0,1)$ is a pseudo-complemented semilattice then by a ${ }^{*}$-congruence on $L$, we shall mean a semilattice congruence $\equiv$ that satisfies the additional condition $x \equiv y \Rightarrow x^{*} \equiv$ $y^{*}$. Now not every ideal of $L$ is the kernel of a *-congruence. The following example, which will be of use throughout the paper, illustrates this simply: let $\mathscr{L}$ be the semilattice whose Hasse diagram is

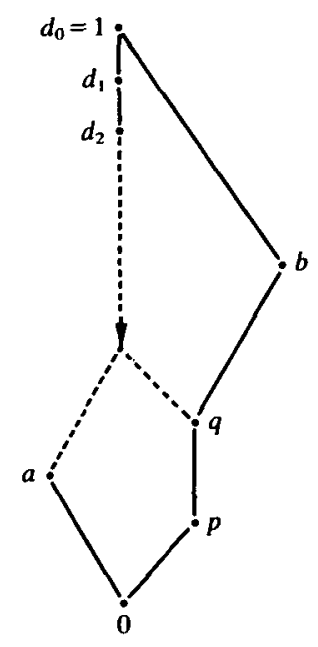

$\mathscr{L}$ is pseudo-complemented; we have $0^{*}=1, a^{*}=b, p^{*}=q^{*}=b^{*}=a, d_{i}^{*}=0$ for $i \geqq 0$. The ideal $I=\{0, a, p, q, b\}$ is not the kernel of $a^{*}$-congruence. For, suppose that $\equiv$ were a *-congruence with kernel $I$; then from $a \equiv b \equiv 0$ we would have $a^{*} \equiv b^{*} \equiv 0^{*}$, i.e. $b \equiv a \equiv 1$, whence the contradiction $1 \equiv a \equiv 0$.

Which ideals, then, of a pseudo-complemented semilattice are kernels of *-congruences? We provide an answer to this question in Section 2. Here we also describe 
the smallest and largest *-congruences having a given kernel ideal. In Section 3 the main object of study is the set of kernel ideals. We show that this is a complete implicative lattice and identify its centre. In Section 4 we consider *-epimorphisms and prove in particular that the lattice of kernel ideals is isomorphic to the lattice of ideals of the skeleton boolean algebra. In Section 5 we turn our attention to filters. Here we show that every filter is the cokernel of a ${ }^{*}$-congruence and investigate a certain type of filter called a ${ }^{*}$-filter. We prove that these filters form a complete lattice that is isomorphic to the lattice of kernel ideals. In Section 6 we consider those ${ }^{*}$-congruences $R$ that are boolean (in that $L / R$ is a boolean algebra) and determine necessary and sufficient conditions for a given kernel ideal (resp. *-filter) to be the kernel (resp. cokernel) of a unique *-congruence. We also describe the smallest boolean congruence that identifies a given pair of elements of $L$. In Section 7 we consider another type of filter, called a $D$-filter, and a determine necessary and sufficient conditions for every $D$-filter to be a *-filter. This happens in particular when the semilattice $L$ is modular.

\section{Kernel ideals and *-ideals}

We refer the reader to (3) for the basic properties of pseudo-complementation. We begin by establishing the following useful result; this does not appear to be in the literature.

Theorem 2.1. If $L$ is a pseudo-complemented semilattice then a semilattice congruence $\equiv$ on $L$ is $a^{*}$-congruence if and only if

$$
x \equiv 0 \Rightarrow x^{*} \equiv 1
$$

Proof. The condition is clearly necessary. Suppose conversely that the condition holds and let $x \equiv y$. Then $0=x \wedge x^{*} \equiv y \wedge x^{*}$ and so $\left(x^{*} \wedge y\right)^{*} \equiv 1$. Using the identity $a \wedge(a \wedge b)^{*}=a \wedge b^{*}$ (see, for example, (3, Theorem 15.1 (viii)), we thus have

$$
x^{*} \equiv x^{*} \wedge\left(x^{*} \wedge y\right)^{*}=x^{*} \wedge y^{*} \text {. }
$$

Similarly we have $y^{*} \equiv x^{*} \wedge y^{*}$, whence $x^{*} \equiv y^{*}$.

Definition. An ideal $I$ of a pseudo-complemented semilattice $L$ will be called a kernel ideal if $\mathrm{I}$ is the kernel of $\mathrm{a}^{*}$-congruence on $L$.

Theorem 2.2. An ideal I of a pseudo-complemented semilattice $L$ is a kernel ideal of $L$ if and only if

$$
i, j \in I \Rightarrow\left(i^{*} \wedge j^{*}\right)^{*} \in I \text {. }
$$

Proof. If $I$ is the kernel of a ${ }^{*}$-congruence $\equiv$ and if $i, j \in I$ then $i^{*} \equiv 1 \equiv j^{*}$ gives $i^{*} \wedge j^{*} \equiv 1$ whence $\left(i^{*} \wedge j^{*}\right)^{*} \in I$.

Conversely, suppose that the condition holds. Consider the relation $R_{I}$ defined on $L$ by

$$
x R_{I} y \Leftrightarrow(\exists i \in I) x \wedge i^{*}=y \wedge i^{*} .
$$

Clearly, $R_{I}$ is both reflexive and symmetric. It is also transitive; for if $i, j \in I$ are such that $x \wedge i^{*}=y \wedge i^{*}$ and $y \wedge j^{*}=z \wedge j^{*}$ then, with $k=\left(i^{*} \wedge j^{*}\right)^{*} \in I$, we have 


$$
\begin{aligned}
x \wedge k^{*}=x \wedge\left(i^{*} \wedge j^{*}\right)^{* *} & =x \wedge i^{*} \wedge j^{*} \\
& =y \wedge i^{*} \wedge j^{*} \\
& =z \wedge i^{*} \wedge j^{*}=z \wedge\left(i^{*} \wedge j^{*}\right)^{* *}=z \wedge k^{*}
\end{aligned}
$$

so that $x R_{I} z$. It is clear that the equivalence relation $R_{I}$ is a semilattice congruence on $L$. Now taking $j=i$ in the condition we obtain $i \in I \Rightarrow i^{* *} \in I$. Thus we have

$$
x R_{I} 0 \Leftrightarrow(\exists i \in I) x \wedge i^{*}=0 \Leftrightarrow(\exists i \in I) x \leqq i^{* *} \Leftrightarrow x \in I,
$$

so the kernel of $R_{I}$ is $I$. Also

$$
x R_{I} 1 \Leftrightarrow(\exists i \in I) x \wedge i^{*}=i^{*} \Leftrightarrow(\exists i \in I) x \geqq i^{*},
$$

so that

$$
x R_{I} 0 \Rightarrow(\exists i \in I) x \wedge i^{*}=0 \Rightarrow(\exists i \in I) i^{*} \leqq x^{*} \Rightarrow x^{*} R_{I} 1 .
$$

It follows by Theorem 2.1 that $R_{I}$ is a *-congruence on $L$. $\square$

Corollary 1. I is a kernel ideal if and only if

(1) $i \in I \Rightarrow i^{* *} \in I$;

(2) $(\forall i, j \in I)(\exists k \in I) i^{*} \wedge j^{*}=k^{*}$.

Proof. The necessity follows from the above proof and the fact that $i^{*} \wedge j^{*}=$ $\left(i^{*} \wedge j^{*}\right)^{* *}$. Conversely, if $(1)$ and (2) hold then clearly $\left(i^{*} \wedge j^{*}\right)^{*}=k^{* *} \in I$.

Corollary 2. A principal ideal $I=x \downarrow$ is a kernel ideal if and only if $x$ is in the skeleton boolean algebra $S(L)$ of $L$.

Proof. $\Rightarrow$ : If $x^{\downarrow}$ is a kernel ideal then by Corollary 1(1) we have $x=x^{* *} \in S(L)$. $x^{\downarrow}$.

$\Leftarrow:$ If $x=x^{* *}$ and $i, j \in x^{\downarrow}$ then $x^{*} \leqq i^{*} \wedge j^{*}$ so $\left(i^{*} \wedge j^{*}\right)^{*} \leqq x^{* *}=x$ whence $\left(i^{*} \wedge j^{*}\right)^{*} \in$

Corollary 3. The following conditions on a pseudo-complemented semilattice $L$ are equivalent:

(1) every ideal of $L$ is a kermel ideal;

(2) every principal ideal of $L$ is a kernel ideal;

(3) $L$ is a boolean algebra.

Proof. (1) $\Rightarrow(2)$ is trivial; (2) $\Rightarrow(3)$ follows from Corollary 2 ; and (3) $\Rightarrow(1)$ follows from the fact that in a boolean algebra $\left(i^{\prime} \wedge j^{\prime}\right)^{\prime}=i \vee j$.

Definition. An ideal $I$ of a pseudo-complemented semilattice $L$ will be called a

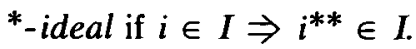

Theorem 2.3. Every kernel ideal is $a^{*}$-ideal. $A$ *-ideal $I$ is a kernel ideal if and only if $\sup _{s(L)}\left\{i^{* *}, j^{* *}\right\}$ belongs to I for all $i, j \in I$. 
Proof. The first statement is immediate from Corollary 1(1) of Theorem 2.2. As for the second, it suffices to observe that in $S(L)$ we have $\sup _{S(L)}\left\{i^{* *}, j^{* *}\right\}=\left(i^{*} \wedge j^{*}\right)^{*}$.

Corollary. If $L$ is a pseudo-complemented distributive lattice then an ideal I of $L$ is a kernel ideal if and only if it is $a^{*}$-ideal.

Proof. In such a lattice we have $(i \vee j)^{*}=i^{*} \wedge j^{*}$ and $\sup _{S(L)}\left\{i^{* *}, j^{* *}\right\}=\left(i^{* *} \vee j^{* *}\right)^{* *}=$ $\left(i^{*} \wedge j^{*}\right)^{*}$.

Theorem 2.4. Let $L$ be a pseudo-complemented semilattice and let I be a kernel ideal of L. Then

(1) the smallest *-congruence on $L$ with kernel I is given by

$$
x R_{I} y \quad \Leftrightarrow \quad(\exists i \in I) x \wedge i^{*}=y \wedge i *
$$

(2) the largest *-congruence on $L$ with kernel $I$ is given by

$$
x R^{I} y \Leftrightarrow\{a \in L ; a \wedge x \in I\}=\{a \in L ; a \wedge y \in I\} .
$$

Proof. (1) It is shown in the proof of Theorem 2.2 that $R_{I}$ is a *-congruence with kernel $I$. If $\equiv$ is any such *-congruence on $L$ then $x R_{I} y$ gives $x \wedge i^{*}=y \wedge i^{*}$ for some $i \in I$ so that, since $i \equiv 0$ and hence $i^{*} \equiv 1$, we have $x \equiv y$.

(2) That $R^{I}$ is the largest semilattice congruence on $L$ with kernel $I$ is proved as in, for example, (1, Theorem 10.5(2)). We show that $R^{I}$ is a ${ }^{*}$-congruence. Clearly, the cokernel of $R^{I}$ is

$$
F=\{x \in L ; a \wedge x \in I \Rightarrow a \in I\} .
$$

Suppose now that $x \in I$. If $a \wedge x^{*} \in I$ then by Theorem 2.2 we have

$$
a \leqq a^{* *} \leqq\left(x^{*} \wedge a^{*}\right)^{*}=\left[x^{*} \wedge\left(a \wedge x^{*}\right)^{*}\right]^{*} \in I
$$

whence $a \in I$ and consequently $x^{*} \in F$. Applying Theorem 2.1 we now see that $R^{I}$ is a *-congruence.

In what follows we shall denote by $G$ the Glivenko congruence $x G y \Leftrightarrow x^{* *}=y^{* *}$. Clearly, $G$ is a ${ }^{*}$-congruence.

Theorem 2.5. If $R$ is $a^{*}$-congruence on $L$ then

$$
x R \vee G y \Leftrightarrow x^{* *} R y^{* *} .
$$

Proof. If $x R \vee G y$ then there exist $a_{0}, \ldots, a_{n} \in L$ such that

$$
x=a_{0} \equiv a_{1} \equiv \ldots \equiv a_{n-1} \equiv a_{n}=y
$$

in which each $\equiv$ signifies either $R$ or $G$. Then

$$
x^{* *}=a_{0}^{* *} \equiv a_{1}^{* *} \equiv \ldots \equiv a_{n-1}^{* *} \equiv a_{n}^{* *}=y^{* *}
$$

where, for $1 \leqq i \leqq n$, either $a_{i-1}^{* *} R a_{i}^{* *}$ or $a_{i-1}^{* *} G a_{i}^{* *}$. Since the latter is equivalent to $a_{i-1}^{* *}=a_{i}^{* *}$ we see that the above chain reduces to $x^{* *} R y^{* *}$.

If, conversely, $x^{* *} R y^{* *}$ then we have the chain 
whence $x R \vee G y$.

$$
x G x^{* *} R y^{* *} G y
$$

We now use Theorem 2.5 to obtain alternative characterisations of $R^{I}$, the largest *-congruence with kernel $I$.

Theorem 2.6. If $I$ is a kernel ideal of $L$ then $R^{I}=R_{I} \vee G$.

Proof. That $I$ is the kernel of $R_{I} \vee G$ follows from the observation that

$$
x R_{I} \vee G 0 \Leftrightarrow x^{* *} R_{I} 0 \Leftrightarrow x^{* *} \in I \Leftrightarrow x \in I .
$$

Suppose now that $R$ is a *-congruence with kernel $I$. If $x R y$ then we have

Since

$$
i=\left[\left(x \wedge y^{*}\right)^{*} \wedge\left(x^{*} \wedge y\right)^{*}\right]^{*} \in I \text {. }
$$

$$
\begin{aligned}
x^{* *} \wedge i^{*} & =x^{* *} \wedge\left[\left(x \wedge y^{*}\right)^{*} \wedge\left(x^{*} \wedge y\right)^{*}\right]^{* *} \\
& =\left[x \wedge\left(x \wedge y^{*}\right)^{*} \wedge\left(x^{*} \wedge y\right)^{*}\right]^{* *} \\
& =\left[x \wedge\left(x \wedge y^{*}\right)^{*}\right]^{* *} \\
& =\left(x \wedge y^{* *}\right)^{* *} \\
& =x^{* *} \wedge y^{* *}
\end{aligned}
$$

and similarly $y^{* *} \wedge i^{*}=x^{* *} \wedge y^{* *}$, it follows that $x^{* *} R_{I} y^{* *}$ and consequently $x R_{I} \vee G y$. Thus $R_{I} \vee G$ is the largest *-congruence with kernel $I$.

Corollary. $x R^{I} y \Leftrightarrow\left[\left(x \wedge y^{*}\right)^{*} \wedge\left(x^{*} \wedge y\right)^{*}\right]^{*} \in I$.

If $I$ is an ideal of $L$ then $I^{o}=\{x \in L ;(\forall i \in I) x \wedge i=0\}$, the set of elements disjoint from $I$, is a kernel ideal; for if $x, y \in I^{o}$ then $i \leqq x^{*}, y^{*}$ gives $\left(x^{*} \wedge y^{*}\right)^{*} \leqq i^{*}$ so $\left(x^{*} \wedge y^{*}\right)^{*} \in I^{o}$.

Theorem 2.7. If $I$ is an ideal of $L$ then

$$
x R^{I^{o}} y \Leftrightarrow x^{* * \downarrow} \cap I=y^{* * \downarrow} \cap I .
$$

Proof. Let $\equiv$ be the equivalence relation defined by

$$
x \equiv y \quad \Leftrightarrow \quad x^{* * \downarrow} \cap I=y^{* * \downarrow} \cap I .
$$

Since $(x \wedge z)^{* * \downarrow}=x^{* * \downarrow} \cap z^{* * \downarrow}$ it is clear that $\equiv$ is compatible with $\wedge$. Now

$$
\begin{aligned}
& x \equiv 0 \quad \Leftrightarrow \quad x^{* * \downarrow} \cap I=0 \quad \Leftrightarrow \quad x^{* *} \in I^{o} \quad \Leftrightarrow \quad x \in I^{o} ; \\
& x \equiv 1 \quad \Leftrightarrow \quad x^{* * \downarrow} \cap I=I \quad \Leftrightarrow \quad I \leqq x^{* * \downarrow} .
\end{aligned}
$$

Thus we have

$$
\begin{aligned}
x \equiv 0 \Rightarrow x \in I^{o} & \Rightarrow(\forall i \in I) i \leqq x^{*} \\
& \Rightarrow I \leqq x^{* \downarrow}=x^{* * * \downarrow} \\
& \Rightarrow x^{*} \equiv 1
\end{aligned}
$$

and so, by Theorem $2.1, \equiv$ is a ${ }^{*}$-congruence with kernel $I^{o}$. 
Suppose now that $x R^{I^{\circ}} y$. Then by the Corollary of Theorem 2.6 we have $\left[\left(x \wedge y^{*}\right)^{*} \wedge\right.$ $\left.\left(x^{*} \wedge y\right)^{*}\right]^{*} \in I^{o}$ whence

$$
(\forall i \in I) \quad\left[\left(x \wedge y^{*}\right)^{*} \wedge\left(x^{*} \wedge y\right)^{*}\right]^{*} \wedge i=0 .
$$

Since $\left[\left(x \wedge y^{*}\right)^{*} \wedge\left(x^{*} \wedge y\right)^{*}\right]^{*} \geqq\left(x \wedge y^{*}\right)^{* *}=x^{* *} \wedge y^{*}$ it follows that, for all $i \in I, x^{* *} \wedge y^{*} \wedge$ $i=0$. If then $i \in x^{* * \downarrow} \cap I$ we have $y^{*} \wedge i=0$ and so $i \leqq y^{* *}$ whence $i \in y^{* * \downarrow} \cap I$. Thus $x^{* * \downarrow} \cap I \subseteq y^{* * \downarrow} \cap I$. Similarly, we can establish the reverse inclusion, whence we obtain $x \equiv y$. Since $R^{I^{\circ}}$ is the largest ${ }^{*}$-congruence with kernel $I^{o}$ it follows that $R^{I^{\circ}}$ and $\equiv$ coincide.

Corollary. If $I$ is a kernel ideal then $R^{I} \wedge R^{I^{\circ}}=G$.

Proof. If $x R^{I} \wedge R^{I^{o}} y$ then by the Corollary of Theorem 2.6 we see that $\left[\left(x \wedge y^{*}\right)^{*} \wedge\right.$ $\left.\left(x^{*} \wedge y\right)^{*}\right]^{*}$ belongs to both $I$ and $I^{o}$, whence it is 0 . It follows that $x^{* *} \wedge y^{*}=0=x^{*} \wedge y^{* *}$ whence $x^{* *} \leqq y^{* * *}$ and $y^{* *} \leqq x^{* *}$ so that $x G y$. Since $R^{I}, R^{I^{\circ}} \geqq G$ by Theorem 2.6 , we deduce that $R^{I} \wedge R^{r^{\circ}}=G$. $\square$.

\section{The lattices of kernel and *-ideals}

It is clear that the set $I^{*}(L)$ of *-ideals of $L$, ordered by set inclusion, is a complete lattice in which the lattice operations are set-theoretic. It is clear that in $I^{*}(L)$ the infinite distributive law

$$
I \cap \underset{\alpha \in A}{\cup} I_{\alpha}=\bigcup_{\alpha \in A}\left(I \cap I_{\alpha}\right)
$$

holds. It follows, therefore, that $I^{*}(L)$ is a complete implicative lattice. An explicit description of residuals in $I^{*}(L)$ is as follows.

Theorem 3.1. If $I, J \in I^{*}(L)$ then

$$
I: J=\left\{x \in L ; x^{* * \downarrow} \cap J \subseteq I\right\} \text {. }
$$

Proof. Given $I, J \in I^{*}(L)$, let $T=\left\{x \in L ; x^{* * \downarrow} \cap J \subseteq I\right\}$. Then $T \neq \varnothing$ since $0 \in T$; and since $x^{* *}=x^{* * * *}$ it follows that $T$ is a ${ }^{*}$-ideal. We now observe that

$$
\begin{aligned}
x \in T \cap J & \Rightarrow x^{* * \downarrow} \cap J \subseteq I, x^{* *} \in J \\
& \Rightarrow x^{* * \downarrow}=x^{* * \downarrow} \cap J \subseteq I \\
& \Rightarrow x \in I
\end{aligned}
$$

so that $T \cap J \subseteq I$. If now $K$ is a ${ }^{*}$-ideal such that $K \cap J \subseteq I$ then

$$
x \in K \Rightarrow x^{* *} \in K \Rightarrow x^{* * \downarrow} \cap J \subseteq K \cap J \subseteq I
$$

and so $K \subseteq T$. Thus $I: J$ exists and is $T$.

Corollary. The centre of $I^{*}(L)$ is trivial.

Proof. If $I \in I^{*}(L)$ is complemented then $I$ has a unique complement, namely $0: I$. Then $L=I \cup 0: I$ gives $1 \in I$ or $1 \in 0: I$. The former gives $I=L$ and the latter gives $I=0$. 
We now note that if $I, J$ are kernel ideals of $L$ then $I \cup J$ is not in general a kernel ideal. For example, in the semilattice $\mathscr{L}$ of Section 1 we have that $a^{\downarrow}, b^{\downarrow}$ are kernel ideals (since $a, b \in S(L)$ ) but $I=a^{\downarrow} \cup b^{\downarrow}$ is not. Indeed, when $L$ is a pseudo-complemented distributive lattice the ideal $I \vee J=\{x \in L ;(\exists i \in I)(\exists j \in J) x \leqq i \vee j\}$ is not in general a kernel ideal; for example, in the pseudo-complemented distributive lattice whose Hasse diagram is

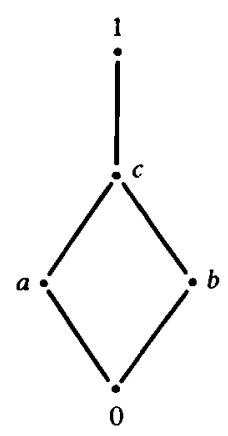

the ideals $a^{\downarrow}, b^{\downarrow}$ are kernel ideals but $a^{\downarrow} \vee b^{\downarrow}=c^{\downarrow}$ is not. In fact we have the following result.

Theorem 3.2. If $L$ is a pseudo-complemented distributive lattice then the following statements are equivalent:

(1) if $I, J$ are kernel ideals of $L$ then so is $I \vee J$;

(2) $l$ is a Stone lattice.

Proof. (1) $\Rightarrow(2)$ : Given $i, j \in L$ we have $i^{* * \downarrow} \vee j^{* * \downarrow}=\left(i^{* *} \vee j^{* *}\right)^{\downarrow}$ so by (1) and Corollary 2 of Theorem 2.2 we see that $i^{* *} \vee j^{* *} \in S(L)$. Thus $S(L)$ is a Stone lattice.

(2) $\Rightarrow(1)$ : If $L$ is a Stone lattice then $(i \vee j)^{* *}=i^{* *} \vee j^{* *}$ for all $i, j \in L$. If now $I, J$ are kernel ideals and $x \in I \vee J$ then $x \leqq i \vee j$ for some $i \in I, j \in J$ whence $x^{* *} \leqq(i \vee j)^{* *}=$ $i^{* *} \vee j^{* *} \in I \vee J$. Thus $x^{* *} \in I \vee J$ and $I \vee J$ is a kernel ideal by the corollary of Theorem 2.4.

Returning to the case of a pseudo-complemented semilattice $L$, we shall denote by $K I(L)$ the set of kernel ideals of $L$.

Theorem 3.3. Ordered by set inclusion, $K I(L)$ forms a complete implicative lattice in which the operations are as follows: if $\left(I_{\lambda}\right)_{\lambda \in \Lambda}$ is a family of kernel ideals of $L$ then

$$
\begin{gathered}
\inf _{K I(L)}\left\{I_{\lambda} ; \lambda \in \Lambda\right\}=\bigcap_{\lambda \in \Lambda} I_{\lambda}, \\
\sup _{K I(L)}\left\{I_{\lambda} ; \lambda \in \Lambda\right\}=\left\{x \in L ;\left(\exists \lambda_{1}, \ldots, \lambda_{n} \in \Lambda\right)\left(\exists x_{i} \in I_{\lambda_{i}}\right) x \leqq\left(\bigwedge_{i=1}^{n} x_{i}^{*}\right)^{*}\right\},
\end{gathered}
$$

and residuals in $K I(L)$ coincide with the corresponding residuals in $I^{*}(L)$. 
Proof. It is clear from Theorem 2.2 that the intersection of any family of kernel ideals is also a kernel ideal, so infima are set-theoretic. Given a family $\left(I_{\lambda}\right)_{\lambda \in \Lambda}$ of kernel ideals, let

$$
U_{\Lambda}=\left\{x \in L ;\left(\exists \lambda_{1}, \ldots, \lambda_{n} \in \Lambda\right)\left(\exists x_{i} \in I_{\lambda_{i}}\right) x \leqq\left(\bigwedge_{i=1}^{n} x_{i}^{*}\right)^{*}\right\}
$$

Then clearly $U_{\Lambda}$ is an ideal of $L$. Now if $x, y \in U_{\Lambda}$ then for suitable $x_{i}$ and $y_{i}$ (some of which may be 0 ) we have $x \leqq\left(\bigwedge_{i=1}^{n} x_{i}^{*}\right)^{*}$ and $y \leqq\left(\bigwedge_{i=1}^{n} y_{i}^{*}\right)^{*}$. By Corollary 1 of Theorem 2.2 there exist $z_{i}$ such that $x_{i}^{*} \wedge y_{i}^{*}=z_{i}^{*}$ for each $i$, so that

$$
\left(x^{*} \wedge y^{*}\right)^{*} \leqq\left[\left(\bigwedge_{i=1}^{n} x_{i}^{*}\right)^{* *} \wedge\left(\bigwedge_{i=1}^{n} y_{i}^{*}\right)^{* *}\right]^{*}=\left(\bigwedge_{i=1}^{n} x_{i}^{*} \wedge \bigwedge_{i=1}^{n} y_{i}^{*}\right)^{*}=\left(\bigwedge_{i=1}^{n} z_{i}^{*}\right)^{*}
$$

whence $\left(x^{*} \wedge y^{*}\right)^{*} \in U_{\Lambda}$. Thus $U_{\Lambda}$ is a kernel ideal. Clearly, $U_{\Lambda}$ contains every $I_{\lambda}$. Suppose now that $J$ is a kernel ideal of $L$ that contains every $I_{\lambda}$. Then if $x_{i} \in I_{\lambda_{i}}$ for $i=1, \ldots, n$ we see by Theorem 2.2 and a simple inductive argument that $\left(\bigwedge_{i=1}^{n} x_{i}^{*}\right)^{*} \in J$, whence $U_{\Lambda} \subseteq J$. This then shows that $K I(L)$ is a complete lattice in which suprema are given by $\sup _{K I(L)}\left\{I_{\lambda} ; \lambda \in \Lambda\right\}=U_{\Lambda}$.

That the complete lattice $K I(L)$ is implicative follows from the fact that the appropriate infinite distributive law holds in $K I(L)$. To see this, let $\left(I_{\lambda}\right)_{\lambda \in \Lambda}$ be a family of kernel ideals and let $I$ be an arbitrary kernel ideal. If $x \in I \sup _{K I(L)}\left\{I_{\lambda} ; \lambda \in \Lambda\right\}$ then, with the above notation, we have

$$
\begin{aligned}
x & \leqq x^{* *} \wedge\left(\bigwedge_{i=1}^{n} x_{i}^{*}\right)^{*} \\
& =x^{* *} \wedge \sup _{S(L)}\left\{x_{i}^{* *} ; 1 \leqq i \leqq n\right\} \\
& =\sup _{S(L)}\left\{x^{* *} \wedge x_{i}^{* *} ; 1 \leqq i \leqq n\right\} \\
& =\left(\bigwedge_{i=1}^{n}\left(x \wedge x_{i}\right)^{*}\right)^{*}
\end{aligned}
$$

whence $x \in \sup _{K I(L)}\left\{I \cap I_{\lambda} ; \lambda \in \Lambda\right\}$.

Finally, to show that residuals in $K I(L)$ coincide with the corresponding residuals in $I^{*}(L)$ it suffices to show that for all $I, J \in K I(L)$ the ${ }^{*}$-ideal $I: J=\left\{x \in L ; x^{* * \downarrow} \cap J \subseteq I\right\}$ belongs to $K I(L)$. Suppose then that $x, y \in I: J$ and let $j \in\left(x^{*} \wedge y^{*}\right)^{* \downarrow} \cap J$. Then $j \wedge x^{*} \wedge$ $y^{*}=0$ gives $j \wedge x^{*} \leqq y^{* *}$ so that $j \wedge x^{*} \in y^{* * \downarrow} \cap J \subseteq I$. But we also have $j \wedge x^{* *} \in$ $x^{* *} \downarrow \cap J \leqq I$. Passing to quotients modulo $R_{I}$ we thus have $j / R_{I} \leqq x^{* *} / R_{I}$ and $j / R_{I} \leqq x^{*} / R_{I}$ from which we deduce that $j / R_{I}=0 / R_{I}$ and hence that $j \in I$. Thus we have $\left(x^{*} \wedge y^{*}\right)^{*} \in$ $I: J$ and so, by Theorem $2: 2, I: J \in K I(L)$.

Theorem 3.4. $J \in K I(L)$ is in the centre of $K I(L)$ if and only if $J$ is principal.

Proof. $\Rightarrow$ : If $J \in K I(L)$ is complemented then $J$ has a unique complement, namely $0: J$. Then $L=\sup _{K I(\alpha)}\{J, 0: J\}$ gives, by Theorem $3.3,1=\left(x^{*} \wedge y^{*}\right)^{*}$ for some $x \in J$ and $y \in 0: J=\left\{z \in L ; z^{* * \downarrow} \cap J=0\right\}$. Since $(\forall j \in J) y^{* *} \wedge j=0$ and hence $(\forall j \in J) j \leqq y^{*}$ we have, for every $j \in J$, 


$$
\begin{aligned}
j^{* *}=j^{* *} \wedge 1 & =j^{* *} \wedge\left(x^{*} \wedge y^{*}\right)^{*} \\
& =\left[j \wedge\left(x^{*} \wedge y^{*}\right)^{*}\right]^{* *} \\
& \leqq\left[j \wedge\left(x^{*} \wedge j\right)^{*}\right]^{* *} \\
& =\left(j \wedge x^{* *}\right)^{* *} \\
& =j^{* *} \wedge x^{* *}
\end{aligned}
$$

whence we obtain $j^{* *}=j^{* *} \wedge x^{* *}$ and so $j \leqq j^{* *} \leqq x^{* *} \in J$. Thus we see that $J=x^{* * \downarrow}$.

$\Leftarrow$ :If $J \in K I(L)$ is principal then $J=x^{* * \downarrow}$ so that

$$
0: J=\left\{y \in L ; y^{* *} \wedge x^{* *}=0\right\}=\{y \in L ; y \wedge x=0\}=x^{* \downarrow} \text {. }
$$

Applying Theorem 3.3 we thus have

$$
\sup _{K I(L)}\{J, 0: J\}=\left\{y \in L ; y \leqq\left(x^{*} \wedge x^{* *}\right)^{*}\right\}=L
$$

whence $\boldsymbol{J}$ is complemented.

Corollary. The centre of $K I(L)$ is isomorphic to $S(L)$.

For the lattice $\mathscr{L}$ of Section 1, the lattices $I^{*}(\mathscr{L})$ and $K I(\mathscr{L})$ are the following:

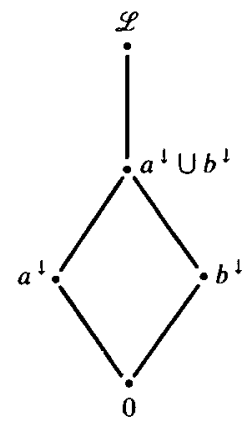

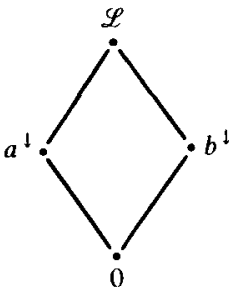

$K I(\mathscr{L})$

\section{4. *-epimorphisms}

$\Gamma^{*}(\mathscr{L})$

If $L, M$ are pseudo-complemented semilattices then a semilattice morphism $f: L \rightarrow M$ will be called a *-morphism if

$$
(\forall x \in L) \quad f\left(x^{*}\right)=[f(x)]^{*} .
$$

If $f: L \rightarrow M$ is a *-morphism and $f(x)=0=f(y)$ then $f\left[\left(x^{*} \wedge y^{*}\right)^{*}\right]=0$, so $\operatorname{Ker} f=$ $\{x \in L ; f(x)=0\}$ is a kernel ideal. In order to distinguish carefully various mappings that we shall consider, we shall find it convenient to use the following notation. Given a mapping $f: E \rightarrow F$ we shall denote by $f^{\rightarrow}: \boldsymbol{P}(E) \rightarrow \boldsymbol{P}(F)$ and $f^{\leftarrow}: \boldsymbol{P}(F) \rightarrow \boldsymbol{P}(E)$ the induced mappings given by the prescriptions

$$
\begin{aligned}
& (\forall X \subseteq E) \quad f(X)=\{f(x) ; x \in X\} ; \\
& (\forall Y \subseteq F) \quad f^{-}(Y)=\{x \in E ; f(x) \in Y\} .
\end{aligned}
$$

Theorem 4.1. If $: L \rightarrow M$ is $a^{*}$-epimorphism then $f^{\rightarrow}$ and $f^{\leftarrow}$ preserve kernel ideals. 
Proof. The proof is an easy application of Theorem 2.2. $\square$

It is immediate from Theorem 4.1 that every *-epimorphism $f: L \rightarrow M$ induces a surjective residuated mapping $f_{K}: K I(L) \rightarrow K I(M)$ described by $I \mapsto f \rightarrow(I)$, the residual of this being the injective mapping $f_{K}: K I(M) \rightarrow K I(L)$ described by $J \mapsto f^{\leftarrow}(J)$.

Theorem 4.2. If $f: L \rightarrow M$ is $a^{*}$-epimorphism then $f_{K}$ is a lattice epimorphism.

Proof. If $x \in f_{K}(I) \cap f_{K}(J)$ then for some $i \in I, j \in J$ we have $x=f(i)=f(j)=$ $f(i \wedge j) \in f_{K}(I \cap J)$ so that $\overrightarrow{f_{K}}(I) \cap f_{K}(J) \subseteq f_{K}(I \cap J)$. The reverse inclusion being obvious, $f_{K}$ preserves infima.

If now $x \in f_{\boldsymbol{K}}\left(\sup _{K I(L)}\{I, J\}\right)$ then by Theorem 3.3 we have $x=f(y)$ where $y \leqq\left(i^{*} \wedge j^{*}\right)^{*}$ for some $i \in I, \quad j \in J$. Then $x \leqq f\left[\left(i^{*} \wedge j^{*}\right)^{*}\right]=\left[(f(i))^{*} \wedge(f(j))^{*}\right]^{*}$ so that $x \in$ $\sup _{K I(L)}\left\{f_{K}(I), f_{K}(J)\right\}$. Thus we have $f_{K}\left(\sup _{K I(L)}\{I, J\}\right) \subseteq \sup _{K I(L)}\left\{f_{K}(I), f_{K}(J)\right\}$. The reverse inclusion being obvious, $f_{K}$ preserves suprema.

When $f$ is a ${ }^{*}$-epimorphism the induced residuated mapping $f_{K}$ is surjective and so is trivially range closed $(1, \$ 13)$. More information is provided by the following result.

Theorem 4.3. If $f: L \rightarrow M$ is $a{ }^{*}$-epimorphism then $f_{K}: K I(L) \rightarrow K I(M)$ is dually range closed.

Proof. By (1, Theorem 13.1*) we have that $f_{K}^{*}$ is dually range closed if and only if, for every $I \in K I(L)$,

$$
f_{K}^{\leftarrow}\left[f_{K}(I)\right]=\sup _{K I(L)}\{I, \operatorname{Ker} f\} .
$$

Since $f_{K}$ and $f_{K}^{\leftarrow}$ are inclusion-preserving, it therefore suffices to show that

$$
f_{K}^{\leftarrow}\left[f_{K}(I)\right] \subseteq \sup _{K I(L)}\{I, \operatorname{Ker} f\} .
$$

Suppose then that $x \in f_{K}\left[f_{K}(I)\right]$. Then for some $i \in I$ we have $f(x)=f(i) \leqq f\left(i^{* *}\right)=$ $\left[f\left(i^{*}\right)\right]^{*}$ so that $f\left(x \wedge i^{*}\right)=f(x) \wedge f\left(i^{*}\right)=0$ whence $x \wedge i^{*} \in \operatorname{Ker} f$. Consequently

$$
x \leqq x^{* *} \leqq\left(i^{*} \wedge x^{*}\right)^{*}=\left[i^{*} \wedge\left(i^{*} \wedge x\right)^{*}\right]^{*}
$$

whence it follows by Theorem 3.3 that $x \in \sup _{K I(L)}\{I, \operatorname{Ker} f\}$.

Corollary. If $K I_{f}(L)$ is the set of kernel ideals of $L$ that contain $\operatorname{Ker} f$ then $K I_{f}(L) \cong$ $K I(M)$.

Proof. By (1, Theorem 13.2) the restriction of $f_{K}$ to $K I_{f}(L)$ is an isomorphism.

Theorem 4.4. $K I(L) \cong I(S(L))$.

Proof. Let $g: L \rightarrow S(L)$ be the Glivenko *-epimorphism, described by $x \mapsto x^{* *}$. Since $S(L)$ is boolean we have, by Corollary 3 of Theorem 2.2 , that $K I(S(L))=I(S(L))$. Since Ker $g=0$ it now follows by the Corollary of Theorem 4.3 that $g_{K}: K I(L) \rightarrow I(S(L))$ is an isomorphism.

Corollary. If $S(L)$ is complete then $K I(L)$ is a Stone lattice. 
We now consider under what conditions the induced residuated mapping $\vec{f}_{K}$ is a *-epimorphism.

Theorem 4.5. If $f: L \rightarrow M$ is $a^{*}$-epimorphism then the following statements are equivalent:

(1) $f_{K}: K I(L) \rightarrow K I(M)$ is a ${ }^{*}$-epimorphism;

(2) Ker $f$ is a principal ideal.

Proof. (1) $\Rightarrow(2)$ : Let $A=\operatorname{Ker} f$; then by (1) we have

$$
f_{K}\left(\sup _{K I(L)}\{A, 0: A\}\right)=f_{K}(0: A)=0: f_{K}(A)=M \text {. }
$$

By the Corollary of Theorem 4.3 it follows that $\sup _{K I(L)}\{a, 0: A\}=L$ whence $A$ is a complemented element of $K I(L)$. That $A$ is principal now follows by Theorem 3.4.

(2) $\Rightarrow$ (1): Clearly, for every $I \in K I(L)$, we have $f_{K}(0: I) \cong 0: f_{K}(I)$ so it suffices, using (2), to establish the reverse inclusion. Now, since $f$ is surjective, we have

and

$$
x \in f_{K}(0: I) \Leftrightarrow x=f(y) \text { where }(\forall i \in I) y \wedge f(i)=0 ;
$$

$$
\begin{aligned}
x \in 0: f_{\kappa}(I) & \Leftrightarrow \quad(\forall i \in I) x \wedge f(i)=0 \\
& \Leftrightarrow \quad x=f(z) \text { where }(\forall i \in I) z \wedge i \in \operatorname{Ker} f .
\end{aligned}
$$

Suppose then that $\operatorname{Ker} f$ is principal. Then by Corollary 2 of Theorem 2.2 we have $\operatorname{Ker} f=t^{* * \downarrow}$ for some $t \in L$. Let $x \in 0: f_{K}(I)$; then $x=f(z)$ where $(\forall i \in I) z \wedge i \in \operatorname{Ker} f$. Now

$$
\left.f(z) \wedge t^{*}\right)=f(z) \wedge[f(t)]^{*}=f(z) \wedge 1=f(z)
$$

and $z \wedge i \wedge t^{*}=0$. Writing $z \wedge t^{*}=a$ we thus have $x=f(a)$ where $(\forall i \in I) a \wedge i=0$, whence $x \in f_{\hat{K}}(0: I)$.

\section{5. *-filters}

Turning our attention to filters, we first prove that, in contrast to the situation concerning ideals, every filter of a pseudo-complemented semilattice is the cokernel of a *-congruence; this extends a known result for implicative semilattices (4).

Theorem 5.1. If $L$ is a pseudo-complemented semilattice and $K$ is a filter of $L$ then the relation $S_{K}$ defined on $L$ by

$$
x S_{K} y \quad \Leftrightarrow \quad(\exists k \in K) x \wedge k=y \wedge k
$$

is $a^{*}$-congruence with cokernel $K$; moreover, $S_{K}$ is the smallest such ${ }^{*}$-congruence.

Proof. It is clear that $S_{K}$ is a semilattice congruence with cokernel $K$. Now if $x S_{K} 0$ then $x \wedge k=0$ for some $k \in K$ whence $k \leqq x^{*}$ gives $x^{*} \in K$ and hence $x^{*} S_{K} 1$. Thus, by Theorem 2.1, $S_{K}$ is a ${ }^{*}$-congruence. Suppose now that $\equiv$ is a ${ }^{*}$-congruence with cokernel $K$. Then if $x S_{K} y$ we have, for some $k \in K, x \equiv x \wedge k=y \wedge k \equiv y$.

We now observe that the condition for filters that is dual to that given in Theorem 2.2, namely $\left(x^{*} \wedge y^{*}\right)^{*} \in K \Rightarrow x, y \in K$, is of no interest; for, if this held then from $\left(x^{*} \wedge\right.$ 
$\left.x^{* *}\right)^{*}=1 \in K$ we would have $x, x^{*} \in K$ whence $0=x \wedge x^{*} \in K$ and $K=L$. However, the condition that is dual to condition (1) of Corollary 1 of Theorem 2.2 is of considerable interest.

Definition. A filter $K$ will be called a *-filter if it is such that

$$
k^{* *} \in K \Rightarrow k \in K
$$

We shall denote the set of *-filters of $L$ by $F^{*}(L)$. The following results, which show how the notion of $a^{*}$-filter arises in a natural way, will allow us to investigate the structure of $F^{*}(L)$.

For every filter $F$ of $L$ let

$$
\alpha(F)=\left\{x \in L ; x^{*} \in F\right\} .
$$

Then $\alpha(F)$ is an ideal of $L$; for if $y \leqq x \in \alpha(F)$ then $y^{*} \geqq x^{*} \in F$ gives $y^{*} \in F$ whence $y \in \alpha(F)$. Moreover, $\alpha(F)$ is a kernel ideal; for if $x, y \in \alpha(F)$ then $x^{*}, y^{*} \in F$ so that $\left(x^{*} \wedge y^{*}\right)^{* *}=x^{*} \wedge y^{*} \in F$ and consequently $\left(x^{*} \wedge y^{*}\right)^{*} \in \alpha(F)$. Denoting by $F(L)$ the lattice of filters of $L$, we can therefore define a mapping $\alpha: F(L) \rightarrow K I(L)$ by $F \mapsto \alpha(F)$.

For every kernel ideal $I$ of $L$ let

$$
\beta(I)=\left\{x \in L ; x^{*} \in I\right\} .
$$

Then $\beta(I)$ is a filter of $L$. In fact, if $y \geqq x \in \beta(I)$ then $y^{*} \geqq x^{*} \in I$ gives $y^{*} \in I$ so that $y \in \beta(I)$; and if $x, y \in \beta(I)$ then $x^{*}, y^{*} \in I$ so that, by Theorem $2.2,(x \wedge y)^{*}=$ $\left(x^{* *} \wedge y^{* *}\right)^{*} \in I$ whence $x \wedge y \in \beta(I)$. We can therefore define a mapping $\beta: K I(L) \rightarrow$ $F(L)$ by $I \mapsto \beta(I)$.

Theorem 5.2. $\alpha$ is residuated with residual map $\beta$.

Proof. For every $F \in F(L)$ we have

$$
\beta[\alpha(F)]=\beta\left\{x \in L ; x^{*} \in F\right\}=\left\{x \in L ; x^{* *} \in F\right\} \supseteqq F ;
$$

and for every $I \in K I(L)$ we have

$$
\alpha[\beta(I)]=\alpha\left\{x \in L ; x^{*} \in I\right\}=\left\{x \in L ; x^{* *} \in I\right\}=I .
$$

Since $\alpha, \beta$ are isotone it follows that $\alpha$ is residuated and that the residual of $\alpha$ is none other than $\beta$.

Corollary. $\beta[\alpha(F)]=F$ if and only if $F$ is $a^{*}$-filter.

It follows from Theorem 5.2 and (1, Theorem 2.7) that $\beta \circ \alpha$ is a closure mapping on the complete lattice $(F(L), \cap, v)$. Using the Corollary of Theorem 5.2 we can therefore assert:

Theorem 5.3. The set $F^{*}(L)$ of *-filters of a pseudo-complemented semilattice $L$, ordered by set inclusion, is a complete lattice in which the lattice operations are as follows: if $\left(F_{\lambda}\right)_{\lambda \in \Lambda}$ is a family of ${ }^{*}$-filters of $L$ then

$$
\inf _{F^{*}(L)}\left\{F_{\lambda} ; \lambda \in \Lambda\right\}=\bigcap_{\lambda \in \Lambda} F_{\lambda}, \quad \sup _{F^{*}(L)}\left\{F_{\lambda} ; \lambda \in \Lambda\right\}=\beta \alpha\left(\bigwedge_{\lambda \in \Lambda} F_{\lambda}\right) \cdot \square
$$


That the lattice $F^{*}(L)$ is also implicative follows immediately from Theorem 3.3 and the following result.

Theorem 5.4. $F^{*}(L) \cong K I(L)$.

Proof. If $I \in K I(L)$ then $\beta(I) \in F^{*}(L)$; for if $x^{* *} \in \beta(I)$ then $x^{*}=x^{* * *} \in I$ so $x \in \beta(I)$. Thus $\beta$ induces an isotone mapping $\hat{\beta}: K I(L) \rightarrow F^{*}(L)$. If we denote by $\hat{\alpha}: F^{*}(L) \rightarrow K I(L)$ the restriction of $\alpha$ to $F^{*}(L)$, the computations in the proof of Theorem 5.2 show that $\hat{\beta} \circ \hat{\alpha}$ and $\hat{\alpha} \circ \hat{\beta}$ are identity mappings. Thus $\hat{\alpha}, \hat{\beta}$ are mutually inverse isomorphisms.

Corollary 1. $F(S(L)) \cong F^{*}(L) \cong K I(L) \cong I(S(L))$.

Proof. Apply Theorem 5.4 to both $L$ and $S(L)$, and combine with Theorem 4.4.

Corollary 2. Suprema in $F^{*}(L)$ are given by

$$
\sup _{F^{*}(L)}\left\{F_{\lambda} ; \lambda \in \Lambda\right\}=\left\{x \in L ; x^{* *} \in \underset{\lambda \in \Lambda}{\bigvee} F_{\lambda}\right\}
$$

Proof. By Theorem 5.4 we have $\sup _{F^{*}(L)}\left\{F_{\lambda} ; \lambda \in \Lambda\right\}=\hat{\beta}\left(\sup _{K I(L)}\left\{\hat{\alpha}\left(F_{\lambda}\right) ; \lambda \in \Lambda\right\}\right)$. Now $x \in \hat{\alpha}\left(F_{\lambda}\right)$ if and only if $x^{*} \in F_{\lambda}$; and, by Theorem 3.3,

$$
\sup _{K I(L)}\left\{\hat{\alpha}\left(F_{\lambda}\right) ; \lambda \in \Lambda\right\}=\left\{x \in L ;\left(\exists \lambda_{1}, \ldots, \lambda_{n} \in \Lambda\right)\left(\exists x_{i}^{*} \in F_{\lambda_{i}}\right) x \leqq\left(\bigwedge_{i=1}^{n} x_{i}^{*}\right)^{*}\right\}
$$

Thus we have

$$
\begin{aligned}
\sup _{F^{*}(L)}\left\{F_{\lambda} ; \lambda \in \Lambda\right\} & =\left\{x \in L ;\left(\exists \lambda_{1}, \ldots, \lambda_{n} \in \Lambda\right)\left(\exists y_{i} \in F_{\lambda_{i}}\right) x^{*} \leqq\left(\bigwedge_{i=1}^{n} y_{i}\right)^{*}\right\} \\
& =\left\{x \in L ;\left(\exists \lambda_{1}, \ldots, \lambda_{n} \in \Lambda\right)\left(\exists y_{i} \in F_{\lambda_{i}}\right) \bigwedge_{i=1}^{n} y_{i} \leqq x^{* *}\right\} \\
& =\left\{x \in L ; x^{* *} \in \underset{\lambda \in \Lambda}{\bigvee} F_{\lambda}\right\} . \square
\end{aligned}
$$

Theorem 5.5. $K$ is $a^{*}$-filter of $L$ if and only if the *-congruence $S_{K} \vee G$ has cokernel $K$. In this case $S_{K} \vee G$ is the largest such ${ }^{*}$-congruence.

Proof. By Theorem 2.5 we have

$$
x S_{K} \vee \dot{G} 1 \Leftrightarrow x^{* *} S_{K} 1 \Leftrightarrow x^{* *} \in K,
$$

from which the first statement follows. Suppose now that $R$ is a *-congruence with cokernel $K$. If $x R y$ then $k=\left(x \wedge y^{*}\right)^{*} \wedge\left(x^{*} \wedge y\right)^{*} \in K$, so

$$
\begin{aligned}
x^{* *} \wedge k & =x^{* *} \wedge\left(x \wedge y^{*}\right)^{*} \wedge\left(x^{*} \wedge y\right)^{*} \\
& =x^{* *} \wedge\left(x \wedge y^{*}\right)^{*} \\
& =\left[x \wedge\left(x \wedge y^{*}\right)^{*}\right]^{* *} \\
& =\left(x \wedge y^{* *}\right)^{* *} \\
& =x^{* *} \wedge y^{* *}
\end{aligned}
$$


and similarly $y^{* *} \wedge k=x^{* *} \wedge y^{* *}$. Thus $x^{* *} S_{K} y^{* *}$ and so $x S_{K} \vee G y$.

Corollary. If $K$ is $a^{*}$-filter then

$$
x S_{K} \vee G y \Leftrightarrow\left(x \wedge y^{*}\right)^{*} \wedge\left(x^{*} \wedge y\right)^{*} \in K . \square
$$

\section{Boolean congruences}

Definition. By a boolean congruence on $L$ we shall mean a *-congruence $R$ such that $L / R$ is boolean.

Theorem 6.1. The following condition on $a{ }^{*}$-congruence $R$ are equivalent:

(1) $R$ is a boolean congruence;

(2) $(\forall x \in L) x R x^{* *}$;

(3) $G \leqq R$;

(4) $R=S_{\text {Cok R }} \vee G$.

Proof. $(1) \Leftrightarrow(2)$ : This is immediate from the fact that in $L / R$ the pseudocomplement of $x / R$ is $x^{*} / R$.

(2) $\Leftrightarrow$ (3): By Theorem 2.5 we have that

$$
G \leqq R \quad \Leftrightarrow \quad R \vee G=R \quad \Leftrightarrow \quad\left(x^{* *} R y^{* *} \quad \Rightarrow \quad x R y\right)
$$

Thus, if $G \leqq R$ we deduce from $x^{* *}=x^{* * * *}$ that $x R x^{* *}$. Conversely, if $x R x^{* *}$ for every $x \in L$ then $x^{* *} R y^{* *}$ implies $x R y$.

(3) $\Leftrightarrow(4)$ : It is clear that (4) $\Rightarrow(3)$. To prove that (3) $\Rightarrow(4)$ it suffices to show that $R \leqq S_{\text {Cok } R} \vee G$. Suppose then that $x R y$. Clearly, $k=\left(x^{*} \wedge y^{* *}\right)^{*} \wedge\left(x^{* *} \wedge y^{*}\right)^{*} \in \operatorname{Cok} R$ and since, as is readily seen, $x^{* *} \wedge k=y^{* *} \wedge k$ it follows that $x^{* *} S_{\text {Cok } R} y^{* *}$ whence $x S_{\text {Cok R }} \vee G y$.

It follows from Theorem 2.6 and 6.1 that if $I$ is a kernel ideal of $L$ then $R^{I}$ is a boolean congruence. We also have the following result.

Theorem 6.2. If $I$ is a kernel ideal of $L$ then the following statements are equivalent:

(1) $R_{I}$ is a boolean congruence;

(2) there is a unique *-congruence with kernel ideal $I$.

Proof. By Theorem 6.1, $R_{I}$ is a boolean congruence if and only if $G \leqq R_{I}$; i.e., by Theorem 2.6, if and only if $R_{I}=R_{I} \vee G=R^{I}$.

Likewise, we see from Theorems 5.5 and 6.1 that if $K$ is a ${ }^{*}$-filter then $S_{K} \vee G$ is a boolean congruence.

Theorem 6.3. If $K$ is. a filter of $L$ then the following statements are equivalent:

(1) $S_{K}$ is a boolean congruence;

(2) $K$ is $a^{*}$-filter and there is a unique *-congruence with cokernel $K$. 
Proof. (1) $\Rightarrow(2)$ : If $G \leqq S_{K}$ then $S_{K}=G \vee S_{K}$ so $G \vee S_{K}$ has cokernel $K$, whence (2) follows by Theorems 5.1 and 5.5.

(2) $\Rightarrow(1)$ : If (2) holds then clearly $S_{K}=G \vee S_{K}$ whence we have (1).

For every $a \in L$ the relation $\theta_{a}$ defined on $L$ by

$$
x \theta_{a} y \Leftrightarrow x \wedge a=y \wedge a
$$

is clearly a semilattice congruence whose kernel is $a^{* \downarrow}$ and whose cokernel is $a^{\uparrow}$. It is immediate from Theorem 2.1 that $\theta_{a}$ is a ${ }^{*}$-congruence.

Theorem 6.4. If $a, b \in L$ then the smallest boolean congruence that identifies $a, b$ is given by

$$
C_{a, b}=\theta_{\left(a \wedge b^{*}\right)^{*}\left(a^{*} \wedge b\right)^{*}} \vee G .
$$

Proof. Since $a^{* *} \wedge\left(a \wedge b^{*}\right)^{*} \wedge\left(a^{*} \wedge b\right)^{*}=a^{* *} \wedge b^{* *}=b^{* *} \wedge\left(a \wedge b^{*}\right)^{*} \wedge\left(a^{*} \wedge b\right)^{*}$ it is immediate by Theorem 2.5 that $a C_{a, b} b$; and, by Theorem 6.1, $C_{a, b}$ is a boolean congruence. Suppose now that $R$ is any boolean congruence on $L$ that identifies $a, b$. In order to show that $C_{a, b} \leqq R$ it suffices, by Theorem 6.1(4), to show that $\theta_{\left(a \wedge b^{*}\right) \wedge\left(a^{*} \wedge b\right)^{*}} \leqq$ $S_{\mathrm{Cok} R}$; and this is immediate from the fact that $a R b$ implies that $\left(a \wedge b^{*}\right)^{*} \wedge\left(a^{*} \wedge b\right)^{*} \in$ Cok R.

\section{D-filters}

Definition. A filter $F$ of a pseudo-complemented semilattice $L$ will be called a $D$-filter if it contains the dense filter $D$.

Theorem 7.1. Every *-filter is a D-filter.

Proof. If $F$ is a ${ }^{*}$-filter and $d \in D$ then $d^{* *}=1 \in F$ gives $d \in F$.

The converse of Theorem 7.1 is not true in general. For example, in the semilattice $\mathscr{L}$ of Section 1 we have $D=\left\{d_{i} ; i \geqq 0\right\}$ and the filter $q^{\uparrow}$ is a $D$-filter that is not a ${ }^{*}$-filter (since $p^{* *}=b \in q^{\uparrow}$ but $p \notin q^{\uparrow}$ ). In fact the lattices $F^{*}(\mathscr{L})$ of *-filters and $D F(\mathscr{L})$ of $D$-filters are

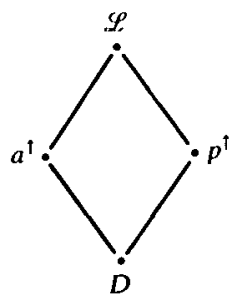

$F^{*}(\mathscr{L})$

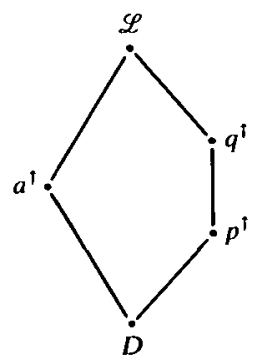

$D F(\mathscr{L})$

Theorem 7.2. For a pseudo-complemented semilattice $L$ the following statements are equivalent:

(1) every $D$-filter is $a *$-filter;

(2) $S_{D}$ is a boolean congruence. 
Proof. (1) $\Rightarrow(2)$ : If (1) holds then for every $x \in L$ the $D$-filter $x^{* * \uparrow} \vee D$ is a ${ }^{*}$-filter. Since $x^{* *} \in x^{* * \uparrow} \vee D$ it follows that $x \in x^{* * \uparrow} \vee D$ whence there exists $d \in D$ such that $x \geqq x^{* *} \wedge d$, so that $x \wedge d=x^{* *} \wedge d$ and hence $x S_{\mathrm{D}} x^{* *}$. That $S_{\mathrm{D}}$ is a boolean congruence now follows by Theorem 6.1 .

(2) $\Rightarrow$ (1): Let $F$ be a $D$-filter. If (2) holds then, by Theorem 6.1, for every $x \in L$ there exists $d \in D$ such that $x \wedge d=x^{* *} \wedge d$. Since $D \cong F$ it follows that

$$
x^{* *} \in F \Rightarrow x \wedge d \in F \Rightarrow x \in F
$$

so that $F$ is a ${ }^{*}$-filter.

Definition. A pseudo-complemented semilattice $L$ will be called $D$-reduced if it satisfies either of the equivalent properties of Theorem 7.2.

Theorem 7.3. Every pseudo-complemented modular semilattice is D-reduced.

Proof. Since $0=x^{* *} \wedge x^{*} \leqq x \leqq x^{* *}$ the modularity gives $x=x^{* *} \wedge d$ for some $d \geqq x^{*}$. Since $d \geqq x$ we have $d^{*} \leqq x^{*} \leqq d \leqq d^{* *}$ whence $d^{*}=0$ and $d \in D$. Consequently we have $x S_{D} x^{* *}$ so that $S_{D}$ is a boolean congruence.

\section{REFERENCES}

(1) T. S. BLYTH and M. F. JANOWITZ Residuation theory (Pergamon Press, 1972).

(2) W. H. CORNiSH, Congruences on distributive pseudo-complemented lattices, Bull. Austral. Math. Soc. 8 (1973), 161-179.

(3) G. GRATZER, Lattice theory: first concepts and distributive lattices (W. H. Freeman, 1971).

(4) W. C. NEMITZ, Implicative semilattices, Trans. Fimer. Math. Soc. 117 (1965), 128-142. 